\title{
ELECTRON CYCLOTRON WAVES *
}

\author{
Egbert Westerhof \\ FOM Institute DIFFER, Dutch Institute for Fundamental Energy Research, \\ PO Box 6336, 5600 HH Eindhoven, The Netherlands, www.differ.nl
}

* These lecture notes form an update of those appearing under the same title in the proceedings of the $10^{\text {th }}$ Carolus Magnus Summer School

\begin{abstract}
This lecture gives an overview of heating and current drive with electron cyclotron waves. We present the main theoretical aspects of wave propagation, wave absorption, and non-inductive current drive, as well as important technical aspects for the application of high power electron cyclotron waves, and the major achievements in their experimental application.
\end{abstract}

\section{INTRODUCTION}

Electron cyclotron waves are electromagnetic waves with a frequency in the range of the electron cyclotron frequency. For a given magnetic field the electron cyclotron frequency is $28 \mathrm{~B}$ [T] GHz. This means that for a typical field of $4 \mathrm{~T}$ or in case of second harmonic resonance and a field of $2 \mathrm{~T}$, we are dealing with frequencies of the order of $100 \mathrm{GHz}$ and, consequently, wavelengths of a few $\mathrm{mm}$. In this frequency range, the waves can be injected from vacuum in the form of wellfocused beams with higher power densities than achieved by any of the other additional heating methods (like neutral beams, ion cyclotron or lower hybrid waves). Moreover, these beams can be injected from steerable mirrors towards different parts of the plasma. Because the coupling of the power to the plasma is the result of a resonant interaction with the electrons, the power deposition in the plasma is localized. This combination of narrow, steerable beams and localized power deposition is unique for electron cyclotron resonance heating $(\mathrm{ECRH})$ and current drive (ECCD). It is also what has given ECRH and ECCD its unique tasks among the other additional heating systems, in particular, for the control of instabilities. Excellent reviews of ECRH and ECCD can be found in Refs [1-4].

\section{THEORETICAL ASPECTS}

\section{II.A. Wave Propagation.}

A basic impression of wave propagation in the electron cyclotron range of frequencies is obtained by analysis of the cold plasma dispersion. The relevant dispersion relation is known as the Appleton-Hartree dispersion relation and is given by

$$
N^{2}=1-\frac{\omega_{p}^{2}}{\omega^{2}} \frac{2\left(\omega^{2}-\omega_{p}^{2}\right)}{2\left(\omega^{2}-\omega_{p}^{2}\right)-\omega_{c}^{2}\left(\sin ^{2} \theta \pm \rho\right)}
$$

with

$$
\rho^{2}=\sin ^{4} \theta+4 \cos ^{2} \theta\left(\frac{\omega^{2}-\omega_{p}^{2}}{\omega-\omega_{c}}\right)^{2},
$$

where $\omega$ is the wave frequency, $\omega_{p} \equiv\left(4 \pi n_{e} e^{2} / m_{e}\right)^{1 / 2}$ the electron plasma frequency, $\omega_{c} \equiv e B / m_{e} c$ the electron cyclotron frequency (defined positive here) and $\theta$ is the angle between the wave vector and the magnetic field. The + and - signs refer to the two possible modes of propagation which are known as the extraordinary or Xmode and ordinary or O-mode, respectively.

Because of toroidal symmetry, $\mathrm{RN}_{\phi}$ is a conserved quantity along the trajectory of wave propagation. This means that the parallel refractive index, $\mathrm{N}_{\|}$, is an approximate constant as well, such that the accessibility of the plasma to electron cyclotron waves can be gleaned from inspection of dispersion curves for the perpendicular wave vector at constant $\mathrm{N}_{\|}$(see Figure 1). Wave cut-offs and resonances can be conveniently defined as cut-offs and resonances of the perpendicular refractive index, i.e. $N_{\perp}=0$ and $N_{\perp}=\infty$, respectively. The O-mode is seen to have a single branch with a cutoff at the plasma frequency $\omega_{\mathrm{p}}$. The X-mode has a right hand, $\omega^{+}$, and a left hand cut-off, $\omega^{-}$, and is split into two branches by an evanescent region between the right hand cut-off and the Upper-Hybrid resonance, $\omega_{\mathrm{UH}}$. The right hand and left hand cut-offs are given by

$$
\omega^{ \pm} \equiv \pm \frac{1}{2} \omega_{c}+\sqrt{\left(\frac{1}{2} \omega_{c}\right)^{2}+\omega_{p}^{2} /\left(1-N_{\|}^{2}\right)},
$$

while the Upper Hybrid resonance is

$$
\omega_{U H} \equiv \sqrt{\omega_{c}^{2}+\omega_{p}^{2}} .
$$

The upper X-mode branch $\omega>\omega^{+}$, which is characterized by faster-than-light phase velocities, is known as the fast X-mode branch, while the other branch limited from above by the Upper Hybrid resonance and from below by the left hand cut-off, $\omega^{-}$, is the slow Xmode. 


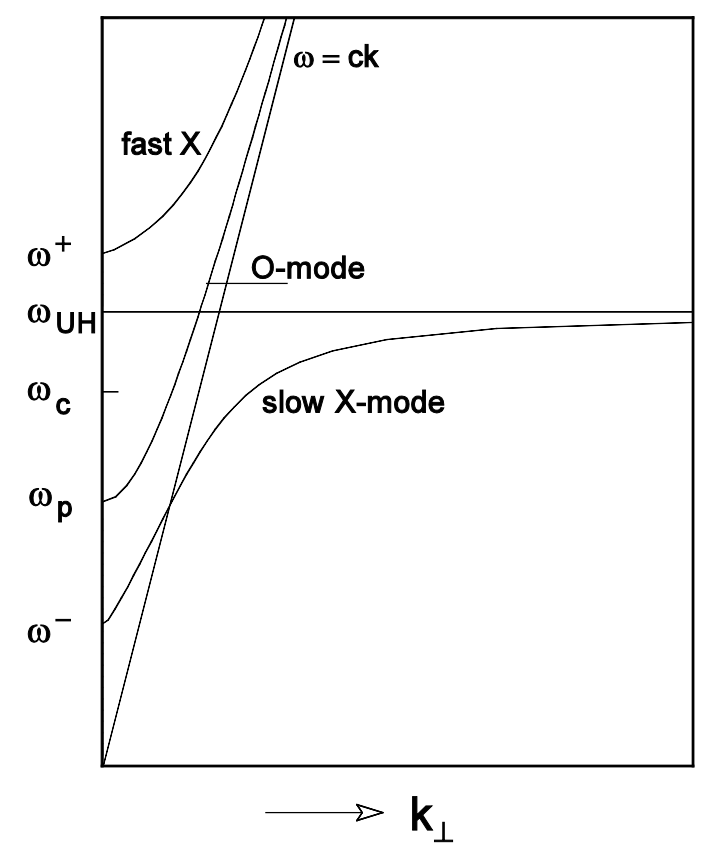

Figure 1: The dispersion diagram of high frequency electromagnetic waves is sketched for an under dense plasma, i.e. $\omega_{p}<\omega_{c}$. The $O$-mode is seen to propagate for $\omega>\omega_{p}$, while the X-mode has two cut-offs $\omega^{+,-}$and a resonance at $\omega_{U H}$.

These wave modes are characterized by their polarization. For perpendicular propagation, the electric field of the O-mode is parallel to the equilibrium magnetic field, and X-mode polarization is perpendicular to the magnetic field. Near the cyclotron resonance, the $\mathrm{X}$-mode attains a large electrostatic contribution, which minimizes the interaction with the right handedly gyrating electrons, i.e. the perpendicular X-mode becomes left handed. Efficient absorption of X-mode at the fundamental resonance requires oblique injection of the waves or a very high plasma temperature.

\section{Wave accessibility}

In a tokamak the magnetic field is approximately inversely proportional to the major radius, $B \propto 1 / R$, and the density usually rises monotonically from the edge to the magnetic axis. This leads to a picture of the wave cut-offs and resonances inside the plasma as sketched in Fig. 2. For the X-mode two cases are shown with either the fundamental or second harmonic resonance inside the plasma. The former case is characterized by the presence of an evanescent layer in between the right hand cut-off and the Upper-hybrid resonance, which shields the fundamental resonance from waves injected from the low field side. Fundamental X-mode heating is only possible with high field side (HFS) launch. The accessibility for second harmonic X-mode and fundamental O-mode is similar. They are in cut-off when either the right hand cut-off $(2 \mathrm{X})$ or plasma frequency (O), exceeds the wave frequency. Thus, waves injected from any direction can reach the resonance as long as the
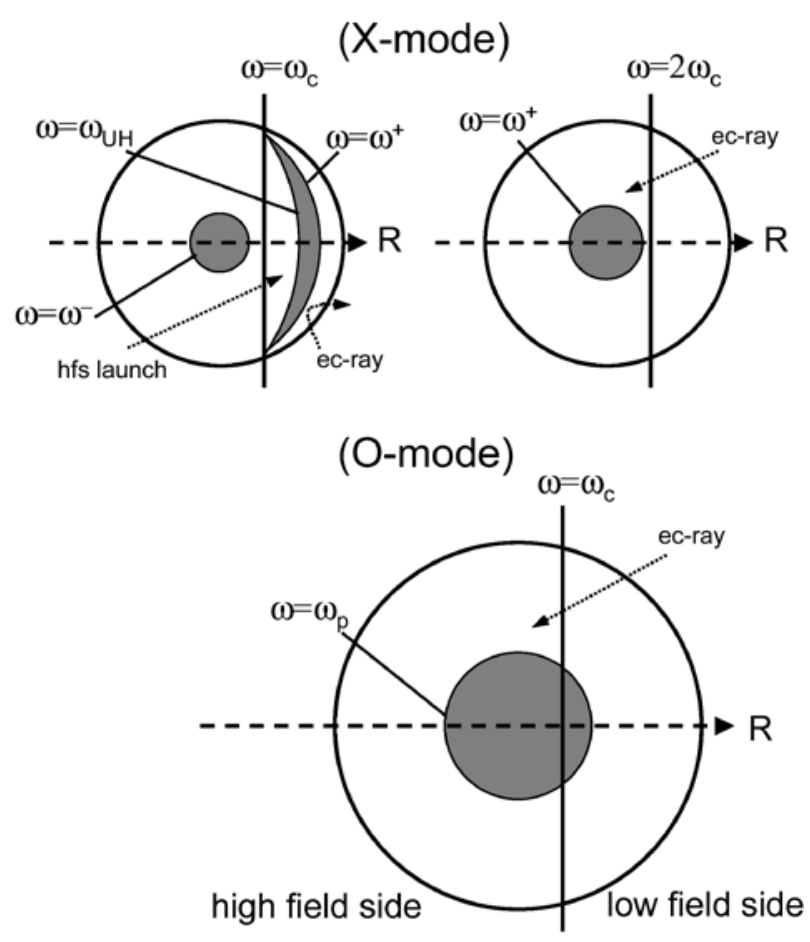

Figure 2: Wave cut-offs and resonances in a poloidal cross section of a tokamak plasma. The upper figure shows two X-mode cases with either the fundamental or second harmonic resonance inside the plasma. The lower figure shows the fundamental O-mode. A high central density is assumed in all cases such that all relevant cut-offs are in the plasma. Gray regions are evanescent. Dotted curves sketch possible wave trajectories. The dashed line indicates the major radius, $R$.

resonance itself is not inside a cut-off region. As densities come close to cut-off, wave refraction will play an important role. Evaluation of actual wave trajectories requires detailed ray-tracing calculations.

\section{II.B. Wave Absorption}

It is straightforward to see how a right handed gyrating electron, satisfying the resonance condition

$$
\omega=\omega_{c} / \gamma+k_{\|} v_{\|}
$$

is continuously accelerated in the vertical direction by a right handed polarized electric field. At the same time one easily sees that for other harmonics or a parallel electric field the interaction always averages to zero unless the wave has a finite perpendicular wave number and the electron a finite Larmor radius $\rho_{\mathrm{e}}$. We will illustrate this by the example of the equation of motion of an electron in a perpendicularly propagating O-mode, $\mathrm{E}_{\|} \cos (\mathrm{k} \perp \mathrm{x}-\omega \mathrm{t})$,

$$
\dot{p}_{\|}=-e E_{\|} \cos \left(k_{\perp} \rho_{e} \sin \left(\omega_{c} t / \gamma\right)-\omega t+\phi\right) .
$$


With the help of the Bessel function identity

$$
\cos z \sin \phi=\sum_{n=-\infty}^{n=\infty} \cos (n \phi) \mathrm{J}_{\mathrm{n}}(z)
$$

this is rewritten in terms of an infinite sum of cyclotron harmonics $n \omega_{\mathrm{c}}$. One obtains,

$$
\dot{p}_{\|}=-e E_{\|} \sum_{n=-\infty}^{n=\infty} \cos \left(n \omega_{c} t / \gamma-\omega t+\phi\right) \mathrm{J}_{n}\left(k_{\perp} \rho_{e}\right),
$$

which shows the possibility of resonant acceleration of electrons satisfying a (harmonic) resonance condition $n \omega_{c} / \gamma=\omega$. Individual particles either gain or lose energy, subject to their phase relative to the wave. Net exchange of energy between wave and plasma depends on the ensemble average over all particles. Just as in the case of Landau damping this depends on the gradient of the distribution function at the cyclotron resonance.

At this stage a side remark must be made as it may appear as if the O-mode leads to an increase of the parallel energy, whereas the wave itself carries no parallel momentum. This is because in our discussion above we have completely neglected the action of the magnetic field that is also associated with the wave. Although it cannot provide a net energy gain it does rotate the momentum vector in such a way, that the net energy gain will be in the perpendicular direction: electron cyclotron absorption leads primarily to an increase in the perpendicular energy of resonant electrons.

A useful quantity is the optical depth $\tau$, which is defined as the integral of the absorption coefficient $\alpha$ along the trajectory $\mathrm{s}$ of the wave, $\tau \equiv \int \alpha d s$. The total absorbed power $\mathrm{P}_{\mathrm{abs}}$ in the plasma is

$$
P_{a b s}=P_{i n j}(1-\exp (-\tau)) .
$$

Optical depths of a plasma slab in which the magnetic field varies as $\mathrm{B} \sim 1 / \mathrm{R}$ are given in Table I. For the Omode, the optical depth is given for perpendicular propagation and harmonics $n=1,2, \ldots$. Similarly for the $\mathrm{X}$-mode and harmonics $\mathrm{n}=2,3, \ldots$. The optical depth for the fundamental harmonic $\mathrm{n}=1$ of the $\mathrm{X}$-mode is given for oblique propagation. In the table, $\mathrm{N}_{O}$ and $\mathrm{N}_{X}$ refer to the refractive indices of the $\mathrm{O}$ - and $\mathrm{X}$-mode, while $v_{t}=\left(k T_{e} / m_{e}\right)^{1 / 2}$ is the thermal electron velocity. Currently, in most ECRH experiments either the fundamental O-mode or second harmonic X-mode is employed. Except near the plasma edge, optical depths of order of one or higher are generally achieved for both fundamental $\mathrm{O}$ - as well as second harmonic X-mode resulting in complete single pass absorption.

\section{II.C. Non-inductive Current Drive}

As described above, EC wave absorption leads mainly to an increase in perpendicular energy of resonant electrons. Nevertheless efficient non-inductive current drive by EC waves is possible. The basic mechanism is best understood as follows. Take an

\begin{tabular}{|c|c|}
\hline \multicolumn{2}{|c|}{ Table I. The optical depth of EC waves ${ }^{1}$} \\
\hline$\tau_{n \geq 1}^{O}(\perp)$ & $\frac{\pi^{2} n^{2(n-1)}}{2^{n-1}(n-1) !} N_{O}^{2 n-1}\left(\frac{\omega_{p}}{\omega_{c}}\right)^{2}\left(\frac{v_{t}}{c}\right)^{2 n} \frac{R}{\lambda}$ \\
\hline$\tau_{n \geq 2}^{X}(\perp)$ & $\begin{array}{c}\frac{\pi^{2} n^{2(n-1)}}{2^{n-1}(n-1) !} A_{n}\left(\frac{\omega_{p}}{\omega_{c}}\right)^{2}\left(\frac{v_{t}}{c}\right)^{2(n-1)} \frac{R}{\lambda} \\
\text { with } A_{n}=N_{X}^{2 n-3}\left(1+\frac{\omega_{p}^{2} / \omega_{c}^{2}}{n\left(n^{2}-1-\omega_{p}^{2} / \omega_{c}^{2}\right)}\right)^{2}\end{array}$ \\
\hline$\tau_{n=1}^{X}(\angle)$ & $\pi^{2} N_{X}^{5}\left(1+\frac{\omega_{p}^{2}}{\omega_{c}^{2}}\right)^{2}\left(\frac{\omega_{c}}{\omega_{p}}\right)^{2}\left(\frac{v_{t}}{c}\right)^{2} \cos ^{2} \theta \frac{R}{\lambda}$ \\
\hline
\end{tabular}
electron with given parallel and perpendicular momentum. This electron will lose its parallel momentum in a typical momentum loss time defined by the collision frequency $v_{\mathrm{m}}$. Now, assume that after interaction with EC waves its perpendicular momentum is increased by a small amount. Again it will lose its parallel momentum in a collision time, but the collision frequency is now decreased since it is proportional to $\sim 1 / v^{3}$. Oblique injection leads to a Doppler shifted resonance and selectively heats electrons moving in one direction, thus generating a net current.

This picture has been formalized by Fisch and Boozer to obtain the current drive efficiency [5]. Suppose an electron is moved from a position $\boldsymbol{v}_{1}$ in velocity space to a position $\boldsymbol{v}_{2}$. As a consequence it contributes an additional amount of current during its subsequent slowing down, which is estimated as

$$
J \equiv \frac{1}{\Delta t} \int^{\Delta t} \mathcal{J}(t) \mathrm{d} t \approx \frac{-e}{\Delta t}\left(\frac{v_{\| 2}}{v_{2}}-\frac{v_{\| 1}}{v_{1}}\right)
$$

where $v_{i}$ are the appropriate collision frequencies for momentum slowing down. The power that has been spent to create this current is $\left(E_{2}-E_{1}\right) / \Delta t$. Substituting differentials for the finite differences, this leads to the current drive efficiency defined as $[4,5]$

$$
\frac{J}{P}=-e \frac{\hat{\boldsymbol{s}} \cdot \nabla_{\mathbf{p}} v_{\|} / v_{\mathrm{m}}}{\hat{\boldsymbol{s}} \cdot \nabla_{\mathbf{p}} p^{2} / 2 m_{e}}
$$

where $\hat{\boldsymbol{s}}$ is the unit vector in the direction of EC wave driven momentum displacement. In case of ECCD, the nominator and denominator must be integrated along the electron cyclotron resonance curve with appropriate weighting for the local (in momentum space) power absorption.

This picture is further complicated by the presence of trapped electrons. During slowing down, an electron may become trapped and, thereby, loose its remaining parallel momentum. This effect can be incorporated in the current drive efficiency (9) by substituting the correction due to trapping in the response function $v_{\|} / v_{\mathrm{m}}$. EC waves can also directly push passing electrons over the trapping boundary thereby destroying their momentum and driving a reversed current, known as the Ohkawa current [6].

The highest current drive efficiencies are expected for deposition of the wave power on fast particles. A large Doppler shift is called for to selectively heat particles with high parallel velocities. Such a Doppler 
shift, of course, requires a corresponding up- or downshift of the wave frequency with respect to the local cyclotron frequency. On this basis, two ECCD scenarios are discerned: the down-shifted scenario with waves injected from the high-field side, and the up-shifted scenario using low-field side injection. In the down shifted scenario, the X-mode is the natural mode of choice, since it has the highest possible absorption for oblique injection. For the up-shifted scenario, either the fundamental $\mathrm{O}$ - or second harmonic $\mathrm{X}$-mode can be used.

The down-shifted scenario is the scenario of choice in smaller tokamaks, where it is difficult to obtain sufficient absorption in the fundamental $\mathrm{O}$ - or second harmonic X-mode. However, in large hot tokamaks the highest ECCD efficiencies are found with up-shifted scenarios. In such devices the optical depth of the fundamental $\mathrm{O}$ - or second harmonic X-mode is sufficient to deposit all power in energetic particles on one side of the resonance.

\section{II.D. Numerical Tools}

\section{Ray- and Beam-tracing codes}

At higher densities and, in particular near cut-off, wave refraction plays an important role and will have to be taken into account in calculations of the power deposition profiles. This is commonly done using raytracing codes in which a wave beam is represented by a large set of individual rays. Each of these rays is then traced through the plasma using the geometric optics approximation [7]. The power absorbed along the ray is evaluated and linear estimates of the non-inductively driven current may be obtained as well.

In many present day experiments focused beams are being used. Near the beam focus simple ray-tracing breaks down. For these conditions, beam tracing-codes have been developed. An example of such a code is the TORBEAM code [8], which describes the propagation through the plasma of a Gaussian wave beam in terms of its central ray trajectory and the evolution of its beam width and curvature.

Most ray- and beam-tracing codes evaluate the trajectories on the basis of cold plasma dispersion only using the full warm plasma dispersion relation to evaluate the power absorbed along these trajectories. However, near electron cyclotron resonance, the warm plasma dispersion and absorption are strongly inhomogeneous in both real and wave vector space. This has important consequences for the wave beam propagation both in terms of its direction [9] as well as for the beam width. Apart from full wave analyses, a proper description of these effects requires new, quasi-optical techniques [10].

Density and magnetic field perturbations due to plasma turbulence also affect wave propagation. As the turbulence is slow compared to the time scale of wave propagation, at any instant in time the wave propagation is determined by the instantaneous plasma state. Averaged over time the net effect of the turbulence can then be obtained from an ensemble average of the different beam realizations. This idea is embodied in a recent in the WKBeam code [11]. In particular, edge turbulence in larger tokamaks like ITER has been found to result in a significant broadening of the wave power deposition profile [12].

\section{Fokker-Planck codes}

When the absorbed power density exceeds the limit of $p_{a b s}\left[\mathrm{MW} / \mathrm{m}^{3}\right] / \mathrm{n}_{\mathrm{e}}^{2}\left[10^{19} / \mathrm{m}^{3}\right]>0.5, \quad$ EC waves will modify the electron momentum distribution function significantly [13]. This has consequences for the local power absorption as well as the non-inductively driven current. The kinetic evolution of the electron momentum distribution function can be described by the bounceaveraged quasi-linear Fokker-Planck equation, which symbolically can be written as [14]

$$
\frac{\partial f_{e}}{\partial t}=\left.\frac{\partial f_{e}}{\partial t}\right|_{\text {collisions }}+\left.\frac{\partial f_{e}}{\partial t}\right|_{\mathrm{ECRH}}+\left.\frac{\partial f_{e}}{\partial t}\right|_{\mathrm{E}_{\|}},
$$

where the distribution function is averaged over the fast gyro and bounce motion of the electrons. This gives an equation for the distribution function on each magnetic surface that is $2 \mathrm{D}$ in momentum space $\left(\mathrm{p}_{\|}, \mathrm{p}_{\perp}\right)$. The Fokker-Planck equation describes the balance between collisions, driving the distribution function back to Maxwellian, the diffusion of resonant particles driven by the waves, as well as the convection caused by a parallel electric field. An additional term can be added to model the anomalous radial transport of electrons. This makes the model 3D and becomes necessary, when the relevant collisional timescale on which a steady state is reached, becomes similar to the time scale for radial transport. Several numerical 3D Fokker-Planck codes are available and have been used to model non-thermal electron generation and current drive under conditions of high power ECRH [14].

\section{TECHNICAL ASPECTS}

\section{III.A. Wave Sources}

The high power required for ECRH and ECCD experiments is provided by gyrotrons [15]. In a gyrotron the waves are generated by leading a weakly relativistic electron beam (typically $\sim 80 \mathrm{kV}$ ) through a resonant cavity in a magnetic field. A magnetic field slightly in excess of $v(\mathrm{GHz}) / 28 \mathrm{~T}$ is required for efficient fundamental interaction. The field must be very stable and is usually generated by a superconducting magnet. The specific (wave guide) mode that is generated in the cavity depends on the magnetic field, the position in the cavity of the electron beam and the size of the cavity. Modern gyrotrons use high order modes (for example, $\mathrm{TE}_{22,6}$ ) to limit dissipation in the cavity. An internal quasi optical mode converter is used to convert the radiation into a Gaussian beam, which is coupled out of the gyrotron.

One of the most critical issues for high power, long pulse gyrotrons is the handling of the power dissipation 
in the various components: in particular, inside the cavity, on the collector where the power of the spent electron beam is being dumped, and in the vacuum window through which the radiation is transmitted. In the latest generation of gyrotrons the dissipation in the collector is reduced by using a depressed collector. This also improves the overall efficiency of the gyrotron to well over $50 \%$ as required by ITER specifications. The material of choice for the vacuum window these days is CVD (Chemical Vapor Deposition) diamond, which combines a very low loss for $\mathrm{mm}$ waves with very high heat conductivity. Long pulse, $1 \mathrm{MW}$ gyrotrons in the 100 to $170 \mathrm{GHz}$ range are now available and $2 \mathrm{MW}$ coaxial gyrotrons are being developed. In addition, multifrequency gyrotrons are now becoming available providing even further flexibility to future ECRH systems.

\section{III.B. Wave Transmission and injection}

Wave transmission from the source to the plasma is commonly achieved by oversized waveguides, quasioptical lines, or a mixture of both. Modern waveguides are now generally corrugated and employ the low loss $\mathrm{HE}_{11}$ mode (hybrid $\mathrm{TE}_{11} / \mathrm{TM}_{11}$ ). This mode couples well to a Gaussian beam and vice versa. The coupling efficiency has a sharp maximum at the appropriate waveguide diameter. Also optical techniques like miter (mirror) bends can be used. In such cases transmission is very efficient with the losses being determined mainly by the bends $(0.25 \%$ to $0.5 \%$ per bend). In case of high power wave transmission or small diameter waveguides, arcing can pose serious problems. In order to avoid arcing, evacuated waveguides have been employed.

In quasi-optical (QO) lines the free space Gaussian beam is transported between mirrors. Though achieving equally high transmission efficiency, QO systems require significantly more space to allow for the expansion of the Gaussian beam between mirrors.

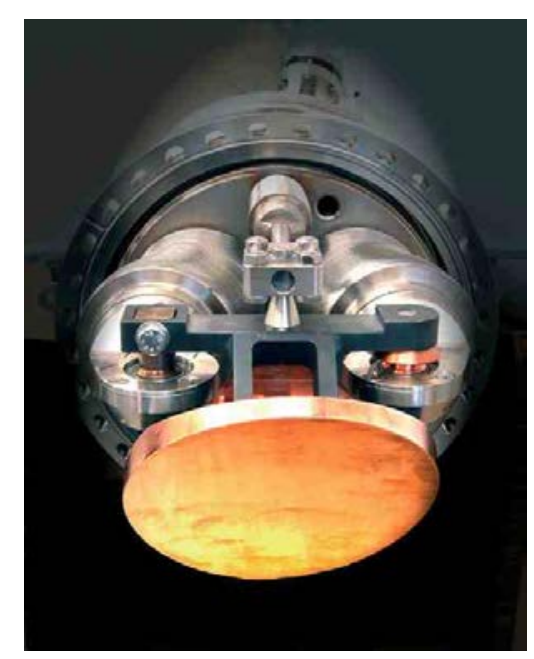

Figure 3: Picture of the launching mirror of the TEXTOR ECRH system. The focusing mirror and the push and pull rods for actuatation of horizontal and vertical rotation are visible.
Typical losses on individual copper mirrors are $0.2 \%$. On most tokamaks, a focused wave beam is finally launched quasi-optically from a steerable mirror. An example of the latter is given in Fig. 3, which shows the launching mirror of the former TEXTOR ECRH system. the TEXTOR ECRH launcher, which was steerable in both the horizontal and vertical planes. Up to date descriptions of modern ECRH systems in use on various tokamaks can be found in Refs [16-20].

\section{EXPERIMENTAL RESULTS}

\section{IV.A. Validation of Propagation and Absorption}

A first step in the experimental verification of theoretical predictions regarding wave propagation and absorption is the measurement of transmitted power. Such measurements have been performed in the electron cyclotron range of frequencies both at low power for diagnostic purposes as well as at high power (see Ref. [2] and references therein). Measurements generally agree well with predictions from ray-tracing as long as refraction is not too strong. It is likely that at high densities, close to cut-off, fluctuations in the plasma can give rise to time varying refraction, which on average leads to a broadening of the wave beam.

The absorption profile can be determined by the initial plasma response following switch-on of ECRH (assuming a steady state at the time of switch on):

$$
p_{\mathrm{ECRH}}(r)=\left.\frac{\partial}{\partial t}\left(\frac{3}{2} n_{e} k T_{e}\right)\right|_{t=t+}
$$

Alternatively, it can be determined by the plasma response at switch-off or by the plasma response to modulated ECRH [21]. The location of the power deposition is generally observed to coincide well with predictions from ray- or beam-tracing $[3,22]$. Several experiments report total amounts of measured power significantly below the amount of power absorbed from the beam according to transmission measurements or calculations. The 'missing power' has been ascribed to fast changes in electron transport or hidden energy reservoirs [2]. In more recent power deposition studies on ASDEX-Upgrade, the full absorbed power could be accounted for in the deposition measurements [22].

\section{IV.B. Validation of Current Drive Predictions}

Measurements of the EC driven current are usually complicated by the presence of other currents like the bootstrap current and, in most cases, the inductive current. The presence of a residual loop voltage also affects the current drive efficiency. Bounce averaged Fokker-Planck code calculations predict significant synergy between ECCD and the loop Voltage in agreement with experiments [23]. Large differences are predicted between co- and counter current drive [24]. The most detailed comparison between experiments and simulations comes from DIII-D. In the analysis of these experiments measurements of the Motional Stark Effect 
are used to determine the internal poloidal magnetic field and from there the current density distribution. Comparing discharges with and without ECCD the driven current is obtained. A series of equilibrium reconstructions is required to obtain the loop voltage at the position of ECCD. That information has been used in a bounce averaged Fokker-Planck code (CQL3D [25]) to simulate the experiments. Such a complete simulation is shown to provide a good fit to the experimental results [26]. Simulations either neglecting the parallel electric field or based on a linear calculation of the driven current lead to unsatisfactory fits. The data set covers co- as well as counter-drive, and a large range of minor radii. In addition, the data covers a wide range in the non-linearity parameter $p_{a b s}\left[\mathrm{MW} / \mathrm{m}^{3}\right] / \mathrm{n}_{\mathrm{e}}^{2}\left[10^{19} / \mathrm{m}^{3}\right]$ with many points near or over the threshold $[3,26]$. The good correspondence of the data and the simulations provides a critical test of the bounce averaged quasilinear Fokker-Planck model including, in particular, trapped particle effects.

Fully non-inductive current drive with ECCD has only been obtained in a limited number of tokamaks. This generally requires special conditions like low density and or low plasma current to maximize on the one hand the EC driven current and on the other hand the bootstrap current fraction. One such example comes from the T-10 tokamak [27]. However, in this case the ECCD pulse was relatively short and a steady state was not reached. More recently, fully non-inductive current drive with ECCD has been obtained in TCV. The discharge could be maintained stable over several current redistribution times provided a sufficiently broad current profile was driven by ECCD [28]. This was achieved by aiming three ECCD beams at different positions in the poloidal cross section.

\section{IV.C. Plasma Heating and Confinement}

In terms of confinement, plasmas heated by ECRH behave in very much the same way as plasmas heated by other additional heating methods such as NBI and ICRH [2]: ECR heated plasmas roughly follow the L-mode scaling. The transition to $\mathrm{H}$-mode is observed at the expected power level or even below. Differences in confinement scaling can be attributed to the strong (central) localization of the ECRH power deposition and the fact that ECRH heats only electrons. The latter two effects also have consequences for the particle transport: the central density some times displays a strong pumpout during ECRH.

In several experiments, very high central electron temperatures of the order of $10 \mathrm{keV}$ have been achieved with central power deposition. In particular, in the presence of an internal transport barrier created by a region of negative or reduced magnetic shear. For example, on TCV [29] this has been achieved by using ECCD to drive some counter-current on axis to establish the negative shear, while on ASDEX Upgrade [30] ECRH has been applied during a preexisting internal transport barrier, established through a programmed current ramp.
The localized nature of the ECRH power deposition makes it an ideal tool for detailed studies of electron transport. One such study has been performed on the RTP tokamak, where a scan of the power deposition over the minor radius revealed a complex response of the plasma with multiple internal transport barriers [31]. Modulation of the ECRH power has become a standard tool for the study of the electron heat diffusivity [21].

\section{IV.D. MHD Stability Control}

The localization of the power deposition also makes ECRH and ECCD ideal tools to control the plasma pressure or current density profiles, which determine the MHD stability of the plasma. Several instabilities are affected by ECRH: sawteeth, tearing modes, ELMs, etc..

Most tokamaks equipped with ECRH report a lengthening of the sawtooth period or complete sawtooth stabilization by ECRH near the sawtooth inversion radius (see, e.g. the early experiments on $\mathrm{T}-10$ [32] or the more recent and more detailed results from TCV [33] and ASDEX Upgrade [34]). The sawtooth crash is triggered when the $m=1, n=1$ internal kink mode is destabilized [35]. The stability threshold of this mode is seen to depend on the shear at the $q=1$ surface. Consequently, changes to the shear at $\mathrm{q}=1$ affected by localized current drive such as ECCD can strongly change the sawtooth period. This has been observed in various experiments and has been modeled in detail for TCV [36]. The dependence of the internal kink stability threshold on the local shear can also be used to derive a simple criterion for the required EC driven current to have a significant effect on the sawtooth period [37]:

$$
I_{c d} \geq 2 I_{q=1}\left(\Delta r_{c d} / r_{q=1}\right)^{2}
$$

where $I_{c d}$ is the non-inductively driven current with a Gaussian width of $\Delta \mathrm{r}_{\mathrm{cd}}$, and $\mathrm{I}_{\mathrm{q}=1}$ is the plasma current inside the $\mathrm{q}=1$ surface, $\mathrm{r}_{\mathrm{q}=1}$. Co-current drive just inside the $\mathrm{q}=1$ surface is found to shorten the sawtooth period, whereas co-current driven just outside the $q=1$ surface will lengthen the sawtooth period. Counter-current drive will have just the opposite effect. A comprehensive review of sawtooth control in provided in Ref. [38].

The control of tearing modes by ECRH and ECCD has received a lot of attention over the years. This stems from the potential threat that these modes pose to tokamak reactors: large tearing modes not only lead to a substantial degradation of plasma confinement, they may also lead to disruption of the plasma [39]. In particular, control of neoclassical tearing modes (NTM) occurring in high $\beta$ tokamak plasmas has been studied [40]. The stability of an (N)TM can be affected in two ways. First, through control of the equilibrium profiles which determine the stability parameter $\Delta^{\prime}$ [41]. Second, by generating additional current, the failing current inside the magnetic island, responsible for its instability, can be replaced resulting in stabilization of the mode [40]. The additional current inside the island can either be generated inductively by heating the island, or driven non-inductively, for example, by ECCD [42]. The 


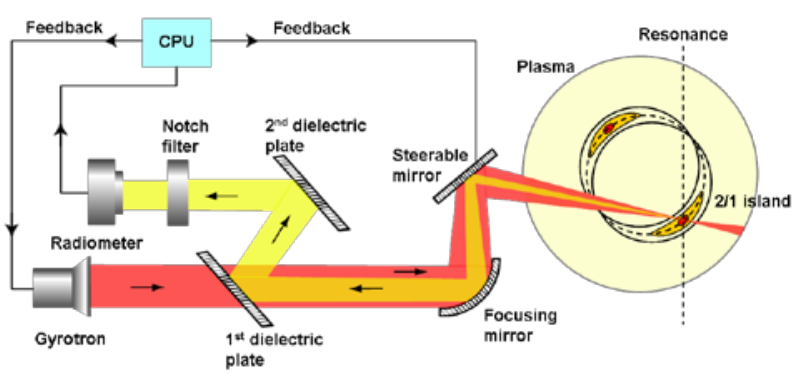

Figure 4: The principle of inline ECE as sensor for feedback controlled EC power deposition. The dielectric plates stand for frequency selective couplers that transmit the high power ECRH waves but reflect the low power ECE at desired frequencies. When the steering mirror is adjusted such that a feature like a 2/1 magnetic island is localized in the sensor spectrum at the actuator (gyrotron) frequency, the power deposition exactly on that feature is ensured.

suppression of NTMs by ECCD has been demonstrated in several experiments [43-46].

In several experiments the NTM suppression was achieved by operation of the ECRH systems under full feedback control $[45,46]$. EC emission (ECE) is used as sensor to detect the location of the NTM with the help of the oscillations due the mode on the ECE signals, and this knowledge is then used to steer the actuator, i.e. the ECRH launcher, in the proper direction. This requires accurate real-time knowledge of the equilibrium and real-time ray tracing to link the sensor derived location to the reference frame of the actuator. To circumvent these latter steps, inline ECE (see Figure 4) has been proposed [47]. In this case the sensing occurs along the reference frame of the actuator, i.e. the gyrotron wave beam. A proof-of-principle inline ECE system was implemented on TEXTOR [48] and used successfully to demonstration tracking and suppression of tearing modes [49].

\section{THE ITER ECRH SYSTEMS}

The international experimental fusion reactor ITER will be equipped with an advanced ECRH system [5052]. According to the ITER design requirements and guidelines this system must have the capability to perform or assist in a number of tasks: (1) heating in order to access $\mathrm{H}$-mode and reach conditions for $\mathrm{Q}>10$ operation, (2) on and off-axis current drive for steady state operation (achieving a current density on axis of $>$ $20 \mathrm{MA} / \mathrm{m}^{2}$ and a total driven current inside $\rho=0.6$ in excess of $1 \mathrm{MA}$ ), (3) stabilization of NTMs by current drive at the $\mathrm{q}=3 / 2$ and $\mathrm{q}=2$ surfaces, (4) wall conditioning, and (5) start-up assist. To (3) should be added the control of sawteeth by current drive at the $q=1$ surface. To perform these tasks an ECRH system has been designed consisting of a total of $24,170 \mathrm{GHz}$ gyrotrons each capable of delivering a power of $1 \mathrm{MW}$, $\mathrm{CW}$. The power will be transferred to the tokamak through evacuated $\mathrm{HE}_{11}$ wave guide using wave guide switches to switch between the alternate upper-port or mid-plane launchers. Due to limited steering capabilities of these launchers, each of them will be used to perform specific tasks. The mid plane launcher is optimized for central heating and current drive, whereas the upper port launcher design is optimized towards the control of NTMs (cf. Fig. 5) and sawteeth. The design work is supported by extensive simulations for the various tasks and by extrapolation from current experiments to ITER [53-55]. Still uncertainties remain. For example, edge density fluctuations have been shown to potentially result in a doubling of the ECCD power deposition profile width near the $\mathrm{q}=1.5$ or 2 rational surfaces, which would correspond to an equivalent increase in the required ECCD power for NTM suppression [12].

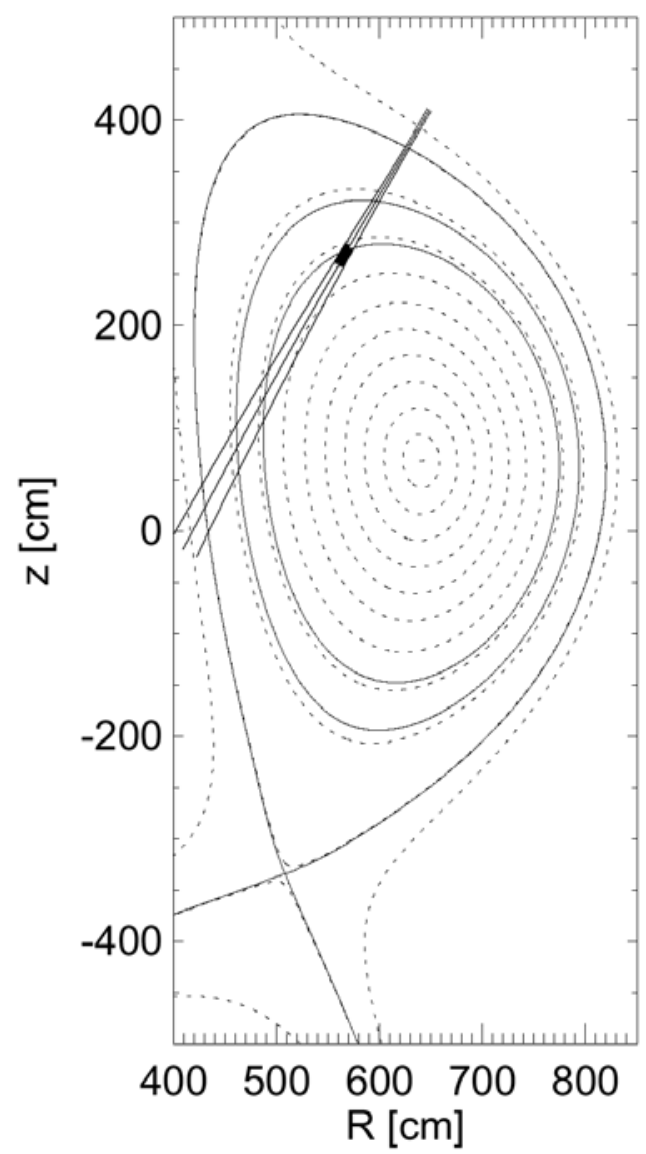

Figure 5: Example of results from of a beam tracing calculation with the TORRBEAM code for a single wave beam coming from an upper launcher, aiming at the $q=3 / 2$ surface. The black region shows the well localized area of power deposition. The dotted curves indicate flux surfaces with some special surfaces indicated by full lines, from the outside going in: the separatrix, the $q=2$, and $q=3 / 2$ surface, respectively.

\section{ACKNOWLEDGMENTS}

This work was carried out with financial support from NWO. 


\section{REFERENCES}

[1] M. BORNATICI, et al. Nucl. Fusion, 23, 1153 (1983).

[2] V. ERCKMANN, and U. GASPARINO, Plasma Phys. Contr. Fusion, 36, 1869 (1994).

[3] R. PRATER, Phys. Plasmas, 11, 2349 (2004).

[4] N.J. FISCH, Rev. Modern Phys. 59175 (1987)

[5] N.J. FISCH, A.H. BOOZER, Phys. Rev. Lett. 45 720 (1980).

[6] T. OHKAWA, Steady state operation of tokamaks by rf heating, General Atomics Report GAA13847 (1976).

[7] R. PRATER, et al., Nucl. Fusion 48035006 (2008).

[8] E. POLI, A.G. PEETERS, G.V. PEREVERZEV, Comput. Phys. Commun. 136, 90 (2001).

[9] M.D. TOKMAN, et al., Nucl. Fusion 43, 1295 (2003).

[10] A.A. BALAKIN, et al., Nuclear Fusion 48, 065003 (2008).

[11] H. WEBER, et al., EPJ Web of Conferences 87, 01002 (2015).

[12] E. POLI, et al., Nucl. Fusion 55 (2015) 013023.

[13] R.W. HARVEY, et al., Phys. Rev. Lett. 62, 426 (1989).

[14] E. WESTERHOF, Proc. of $9^{\text {th }}$ Joint Workshop on ECE and ECH, Borrego Springs, California, 1995, Ed. J. Lohr, World Scientific, Singapore, p. 3 (1995).

[15] G.S. NUSINOVICH, M.K.A. THUMM, M.I. PETELIN, J. Infrared Milli Terahz Waves 35 (2014) 325.

[16] T.P. GOODMAN, et al., Proc. $19^{\text {th }}$ Symp. Fusion Technology, Lisbon, 16-20 September 1996, Ed. C. Varandas, F. Serra (North-Holland, Amsterdam, 1997), Vol. 1 pp. 565 (1996).

[17] F. LEUTERER, et al., Nucl. Fusion 43, 1329 (2003).

[18] R.W. CALLIS, et al., Nucl. Fusion 43, 1501 (2003).

[19] Y. IKEDA, et al., Fus. Eng. Design 53, 351 (2001).

[20] E. WESTERHOF, et al., Nucl. Fusion 43, 1371 (2003).

[21] G.M.D. HOGEWEIJ, Transport studies using perturbative experiments, these proc. (2012).

[22] K.K. KIROV, et al., Plasma Phys. Controlled Fusion 44, 2583 (2002).

[23] R.A. JAMES, et al., Phys. Rev. A 88, 8783 (1992).

[24] A. MERKULOV, et al., Phys. Plasmas 14, 052508 (2007).

[25] R.W. HARVEY, M.G. McCOY, Proc. IAEA TCM Advances in simulations and modeling of thermonuclear plasmas, Montreal, Canada, 1992 (IAEA, Vienna, 1993) p.498.

[26] C.C. PETTY, et al., Nucl. Fusion 42, 1366 (2002).

[27] V.V. ALIKAEV, et al., Nucl. Fusion 32, 1811 (1992).
[28] O. SAUTER, et al., Phys. Rev. Lett. 84, 3322 (2000).

[29] Z.A. PIETRZYK, et al., Phys. Rev. Lett. 86, 1530 (2001).

[30] S. GÜNTER, et al., Phys. Rev. Lett. 84, 3097 (2000).

[31] N.J. LOPES CARDOZO, et al., Plasma Phys. Control. Fusion 39 B303 (1997)

[32] R.M.J. SILLEN, et al., Nucl. Fusion 26, 303 (1986).

[33] Z.A. PIETRZYK, et al., Nucl. Fusion 39, 587 (1999).

[34] A. MÜCK, et al., Plasma Phys. Control. Fusion 471633 (2005).

[35] F. PORCELLI, et al., Plasma Phys. Control. Fusion 38, 2163 (1996).

[36] C. ANGIONI, et al., Nucl. Fusion 43, 455 (2003)

[37] A. MERKULOV, et al., Theory of Fusion Plasmas, Proc. Joint Varenna-Lausanne Int. Workshop, Varenna, Italy, August 30 - September 3 2004, J.W. Connor, O. Sauter, and E. Sindoni (Editors), Societa Italiana di Fisica, Bologna, 2004, p. 279.

[38] I.T. CHAPMAN, et al., Plasma Phys. Control. Fusion 49 (2007) B385-B394.

[39] H.R. KOSLOWSKI, Operational limits and limiting instabilities in tokamak machines, these proceedings (2015)

[40] R.J. LA HAYE, Phys. Plasmas 13, 055501 (2006).

[41] E. WESTERHOF, Nucl. Fusion 301143 (1990)

[42] C.C. HEGNA, J.D. CALLEN, Phys. Plasmas 4, 2940 (1997).

[43] G. GANTENBEIN, et al., Phys. Rev. Lett. 85, 1242 (2000).

[44] C.C. PETTY, et al., Nucl. Fusion 44, 243 (2004).

[45] A. ISAYAMA, et al., Nucl. Fusion 43, 1272 (2003).

[46] F. FELICI, et al., Nucl. Fusion 52 (2012) 074001.

[47] E. WESTERHOF, et al., $13^{\text {th }}$ Joint Workshop on ECE \& ECRH (17-20 May 2004), ed. A. Litvak (Nizhny Novgorod, Russia: IAP/RAS) p. 357 (2005).

[48] J.W. OOSTERBEEK, et al., Rev. Sci. Instruments 79, 093503 (2008).

[49] B.A. HENNEN, et al., Plasma Phys. Controlled Fusion 52, 104006 (2010)

[50] M.A. HENDERSON, et al., Nucl. Fusion 48, 054013 (2008).

[51] K. TAKAHASHI, et al., Fusion Sci. Technol. 47, 1 (2005)

[52] M. HENDERSON, et al., Phys. Plasmas 22, 021808 (2015).

[53] D. FARINA, et al., Phys. Plasmas 21, 061504 (2014).

[54] R.J. LA HAYE, et al., Nucl. Fusion 46, 451 (2006).

[55] I.T. CHAPMAN, et al., Nucl. Fusion 53 (2013) 066001. 